\title{
高熵陶瓷 $\left(\mathrm{Zr}_{1 / 7} \mathrm{Hf}_{1 / 7} \mathrm{Ce}_{1 / 7} \mathrm{Y}_{2 / 7} \mathrm{La}_{2 / 7}\right) \mathrm{O}_{2-\delta}$ 的制备及烧结行为
}

\author{
张丰年, 郭猛, 苗洋, 高峰, 成楚飞, 程富豪, 刘宇峰 \\ (太原理工大学 材料科学与工程学院, 太原 030024)
}

\begin{abstract}
摘 要: 近年来, 不同体系的高摘陶瓷迅猛发展, 但萤石结构高摘氧化物仍处于研发初期。本研究采用机械球磨和 常压烧结的方法合成一种新型高熵萤石氧化物, 利用 XRD, SEM, TG-DSC 和可视化形变分析仪研究了陶瓷的物相 转变、表面形貌以及烧结行为。研究结果表明, $\left(\mathrm{Zr}_{1 / 7} \mathrm{Hf}_{1 / 7} \mathrm{Ce}_{1 / 7} \mathrm{Y}_{2 / 7} \mathrm{La}_{2 / 7}\right) \mathrm{O}_{2-\delta}$ 是一种非等摩尔的“高熵”陶瓷, 其内部 各元素分布均匀。湿法球磨和干法球磨制备的粉末结构和粒度均有所不同, 单相转变温度为 1600 和 $1300{ }^{\circ} \mathrm{C}$, 圆盘 状坯体在 $1600{ }^{\circ} \mathrm{C}$ 保温 $1 \mathrm{~h}$, 尺寸收缩率约为 8.5\%和 17.8\%, 致密度分别为 $82.25 \%$ 和 $93.23 \%$, 说明球磨工艺是影响 高摘陶瓷烧结的重要因素。此外, 常压烧结制备 $\left(\mathrm{Zr}_{1 / 7} \mathrm{Hf}_{1 / 7} \mathrm{Ce}_{1 / 7} \mathrm{Y}_{2 / 7} \mathrm{La}_{2 / 7}\right) \mathrm{O}_{2-\delta}$ 时, 在 $1300 ~ 1600{ }^{\circ} \mathrm{C}$ 应适当减缓升温速 率并延长保温时间，避免生坏收缩开裂，并提高陶瓷致密度。
\end{abstract}

关 键 词: 高熵氧化物; 非等摩尔; 萤石结构; 球磨工艺

中图分类号: TB332 文献标识码: A

\section{Preparation and Sintering Behavior of High-entropy Ceramic $\left(\mathrm{Zr}_{1 / 7} \mathrm{Hf}_{1 / 7} \mathrm{Ce}_{1 / 7} \mathbf{Y}_{2 / 7} \mathrm{La}_{2 / 7}\right) \mathbf{O}_{2-\delta}$}

ZHANG Fengnian, GUO Meng, MIAO Yang, GAO Feng, CHENG Chufei, CHENG Fuhao, LIU Yufeng (College of Materials Science and Engineering, Taiyuan University of Technology, Taiyuan 030024, China)

\begin{abstract}
High entropy ceramics (HECs) typically refer to a kind of solid solution consisting of five or more principal elements. In recent years, different kinds of HECs have developed rapidly whereas high entropy oxides with fluorite structure are still in their nascent state. In this study, a new type of high entropy fluorite oxide was successfully synthesized using mechanical ball-milling followed by conventional sintering. Phase transformation, surface morphology and sintering behavior of ceramic were characterized. The results demonstrate that $\left(\mathrm{Zr}_{1 / 7} \mathrm{Hf}_{1 / 7} \mathrm{Ce}_{1 / 7} \mathrm{Y}_{2 / 7} \mathrm{La}_{2 / 7}\right) \mathrm{O}_{2-\delta}$ is a non-equimolar high entropy ceramic in which all elements distribute homogenously. The powders derived from wet-milling or dry-milling possess different crystal structure and particle size. Raw materials transform into single-phase structure at $1600{ }^{\circ} \mathrm{C}$ and $1300{ }^{\circ} \mathrm{C}$, respectively. After dwelling at $1600{ }^{\circ} \mathrm{C}$ for $1 \mathrm{~h}$, the shrinkage of two discs are $8.5 \%$ and $17.8 \%$, approximately, at the same time, the relative densities are 82.25\% and $93.23 \%$. These miscellaneous distinctions indicate that ball-milling process is a crucial factor affecting sinterability of HECs. In order to avoid cracks caused by rapid shrinkage and improve density of ceramic, it is vital to decrease heating rate and prolong dwell time in the range of $1300-1600{ }^{\circ} \mathrm{C}$ as preparing $\left(\mathrm{Zr}_{1 / 7} \mathrm{Hf}_{1 / 7} \mathrm{Ce}_{1 / 7} \mathrm{Y}_{2 / 7} \mathrm{La}_{2 / 7}\right) \mathrm{O}_{2-\delta}$
\end{abstract}

收稿日期: 2020-07-06; 收到修改稿日期：2020-08-28; 网络出版日期：2020-10-19

基金项目: 国家自然科学基金青年基金(51802213); 山西省应用基础研究计划面上青年基金(201901D211118) National Natural Science Foundation of China (51802213); Program of Applied Basic Research Program of Shanxi Province (201901D211118)

作者简介: 张丰年(1998-), 男, 硕士研究生. E-mail: zhangfn1998@163.com

ZHANG Fengnian(1998-), male, Master candidate. E-mail: zhangfn1998@163.com

通信作者: 苗 洋, 副教授. E-mail: miaoyang@tyut.edu.cn MIAO Yang, associate professor. E-mail: miaoyang@tyut.edu.cn 
by conventional sintering.

Key words: high entropy oxide; non-equimolar; fluorite structure; ball-milling process

高熵合金(High entropy alloys, HEAs)一般是将 多种近原子比的合金元素固溶在一起，每种元素的 含量在 $5 \%$ 35\%, 最终形成一种单相结构 ${ }^{[1]}$ 。与传统 的二主元合金相比，高熵合金具有优异的物理化学 性能, 如高强度 ${ }^{[2]}$ 、耐腐蚀 ${ }^{[3]}$ 和良好的热稳定性 ${ }^{[4]}$, 主要得益于高熵材料的四大效应：高熵效应，迟滞 扩散效应，晶格畸变效应以及鸡尾酒效应。作为一 个相对成熟的体系，高熵合金为其他高熵材料的发 展奠定了坚实的基础。

2015 年, Rost 等 ${ }^{[5]}$ 首次将高熵概念引入到高熵 氧化物(High entropy oxides, HEOs)领域，在 $1000{ }^{\circ} \mathrm{C}$ 成功合成了具有单一氯化钠结构的 $\left(\mathrm{Mg}_{0.2} \mathrm{Co}_{0.2} \mathrm{Ni}_{0.2} \mathrm{Cu}_{0.2} \mathrm{Zn}_{0.2}\right) \mathrm{O}$ 陶瓷。此后, 相继研发了 各种高熵陶瓷, 如高熵碳化物 ${ }^{[6]}$ 、氮化物 ${ }^{[7]}$ 、硼化 物 $^{[8]}$ 、硅化物 ${ }^{[9]}$ 、氟化物 ${ }^{[10]}$, 还有硫化物 ${ }^{[11]}$ 。高熵 陶瓷的合成并不局限于传统的固相法, 先进的合成 方法如机械化学法 ${ }^{[7]}$ 、喷雾热分解法 ${ }^{[12]}$ 、溶液燃烧 法 $^{[13]}$ 以及磁控溅射法 ${ }^{[14]}$ 均适用于高熵陶瓷, 从而促 进高熵陶瓷的迅猛发展 ${ }^{[15]}$ 。作为高熵陶瓷的分支, 高熵氧化物陶瓷演绎出丰富的晶体结构, 以 $\mathrm{Mg}$ 、 $\mathrm{Co} 、 \mathrm{Ni}$ 为主要元素的氯化钠结构高摘氧化物是发展


电常数 ${ }^{[17]}$ 、反铁磁性 ${ }^{[18]}$ 、锂离子超导 ${ }^{[19]}$, 还可用作 催化剂载体 ${ }^{[20-21]}$, 具有十分广泛的用途。钙钛矿结 构 $\left(\mathrm{ABO}_{3}\right)$ 的高熵陶瓷可以在 A-site 和 B-site 同时掺 杂, 形成十组分单相氧化物 ${ }^{[22]}$ 。这类陶瓷具有电热 效应 ${ }^{[23]}$ 、铁电极化储能效应 ${ }^{[24]}$, 可用作析氢催化 ${ }^{[25]}$ 和析氧催化 ${ }^{[26]}$ 载体。高熵陶瓷还可制备成尖晶石和 反尖晶石结构 ${ }^{[27]}$, 它们具有优异的铁磁性、储锂性 能和电催化性能 ${ }^{[28-30]}$ 。另外, 一系列的高熵烧绿石 结构 $\mathrm{Re}_{2} \mathrm{Zr}_{2} \mathrm{O}_{7}{ }^{[31]}$ 、石榴石结构 $\mathrm{Re}_{3} \mathrm{Al}_{5} \mathrm{O}_{12}{ }^{[32]}$ 、含磷 氧化物 ${ }^{[33]}$ 和含硅氧化物 ${ }^{[34]}$ 都具有较低的热导率, 可用作热绝缘材料。磁铅矿结构高熵陶瓷具有铁磁 性、稳定的介电常数和磁导率等性质 ${ }^{[35-36]}$, 目前关 于它们的报道比较少。

萤石结构是一种特殊的面心立方结构, 阳离子 位于晶胞的八个角顶和六个面心，阴离子填充于八 个小立方体的中心。立方氧化锆和氧化铈都具有萤 石结构, 晶胞中含有大量的空隙, 是经典的氧离子 导体 ${ }^{[37]}$ 。近几年, 萤石结构的高熵氧化物也得到了 迅速发展, 主要集中于新成分和新方法的探索 ${ }^{[38-39]}$, 它具有窄带隙, 高电导和低热导等特点 ${ }^{[40-41]}$, 有很
大的发展潜能和应用前景。

本研究合成了一种具有萤石结构的新型非等摩 尔高熵陶瓷 $\left(\mathrm{Zr}_{1 / 7} \mathrm{Hf}_{1 / 7} \mathrm{Ce}_{1 / 7} \mathrm{Y}_{2 / 7} \mathrm{La}_{2 / 7}\right) \mathrm{O}_{2-\delta}$, 研究了陶 瓷升温过程中的物相转变情况, 并综合对比研究了 球磨工艺对高熵陶瓷烧结的影响。

\section{1 实验方法}

\section{1 生料粉末的制备}

采用商业化的氧化物作为原料, 其中 $\mathrm{ZrO}_{2}$ 、 $\mathrm{Y}_{2} \mathrm{O}_{3}$ 和 $\mathrm{La}_{2} \mathrm{O}_{3}$ 均采购于邦瑞新材料科技有限公司, 粒度 $10 \mu \mathrm{m}, 99.9 \%$; $\mathrm{HfO}_{2}$ 采购于河北中越金属材料 科技有限公司，粒度 1 3 $\mu \mathrm{m}, 99.9 \% ; \mathrm{CeO}_{2}$ 采购于上 海阿拉丁生化科技股份有限公司，99.9\%。 $\mathrm{ZrO}_{2}$ 、 $\mathrm{HfO}_{2} 、 \mathrm{CeO}_{2} 、 \mathrm{Y}_{2} \mathrm{O}_{3} 、 \mathrm{La}_{2} \mathrm{O}_{3}$ 按照摩尔比 $1: 1: 1: 1: 1$, 用电子天平准确称取各氧化物原料(氧化物的晶体 学性质见表 1), 在行星式球磨机 QM3SP2(南京南大 仪器)中, 用玛瑙罐+玛瑙球对原料进行球磨, 每 $30 \mathrm{~min}$ 停止 $10 \mathrm{~min}$ 。实验按照不同的球磨工艺对相 同的原料进行球磨, 如表 2 所示, 得到的生料粉末 分别命名为 $\mathrm{A}$ 和 $\mathrm{B}$ 。

\section{2 陶瓷块体的烧结}

称取 $1 \mathrm{~g}$ 左右的生料粉末装入直径 $10 \mathrm{~mm}$ 的圆 柱钢模, 在 $200 \mathrm{MPa}$ 的压力下保压 $3 \mathrm{~min}$ 冷压成型。 利用 KSL-1700x(合肥科晶)箱式炉, 按照一定的升 温程序将陶瓷坏体加热至目标温度, 保温 $1 \mathrm{~h}$ 后程 序降温。随后用金刚石研磨亳抛光陶瓷表面, 用于 物相结构测试和微观组织表征。烧结后陶瓷的化学 式为 $\left(\mathrm{Zr}_{1 / 7} \mathrm{Hf}_{1 / 7} \mathrm{Ce}_{1 / 7} \mathrm{Y}_{2 / 7} \mathrm{La}_{2 / 7}\right) \mathrm{O}_{2-\delta}$ ( 以下缩写为 ZHCYLO)。

\section{表 1 氧化物的晶体结构、空间群及对应 阳离子配位数和半径 ${ }^{[42]}$}

Table 1 Crystal structures, space groups (number), cation coordination numbers $(\mathrm{CN})$ and corresponding cationic radii $\left(r_{c}\right)$ of selected oxides ${ }^{[42]}$

\begin{tabular}{cllll}
\hline Oxides & $\begin{array}{c}\text { Crystal } \\
\text { structure }\end{array}$ & $\begin{array}{c}\text { Space group } \\
\text { (number) }\end{array}$ & $\mathrm{CN}$ & $r_{\mathrm{c}} / \mathrm{nm}$ \\
\hline $\mathrm{ZrO}_{2}$ & Cubic & Fm-3m (225) & 8 & 0.084 \\
$\mathrm{HfO}_{2}$ & Monoclinic & P21/c (14) & 8 & 0.083 \\
$\mathrm{CeO}_{2}$ & Fluorite & Fm-3m (225) & 8 & 0.097 \\
$\mathrm{Y}_{2} \mathrm{O}_{3}$ & Bixbyite & Ia-3 (206) & 6 & 0.090 \\
$\mathrm{La}_{2} \mathrm{O}_{3}$ & Trigonal & P-3m (164) & 6 & 0.1032 \\
\hline
\end{tabular}


表 2 制备生料的工艺、球料比、转速、时间及其他参数

Table 2 Process, ball-to-powder-ratio (BPR), speed, time and others for raw material preparation

Sample Process BPR $\begin{gathered}\text { Speed Time } \\ /\left(\mathrm{r} \cdot \mathrm{min}^{-1}\right)\end{gathered} / \mathrm{h}$ Disperser Desiccation Energy

\begin{tabular}{ccccccc} 
A $\begin{array}{c}\text { Wet } \\
\text { milling }\end{array}: 1$ & 250 & 6 & Ethanol & $\begin{array}{c}60{ }^{\circ} \mathrm{C} \\
/ 24 \mathrm{~h}\end{array}$ & Low \\
B $\begin{array}{c}\text { Dry } \\
\text { milling }\end{array} 10: 1$ & 400 & 40 & - & - & High \\
\hline
\end{tabular}

\section{3 微观结构表征和性能测试}

采用日本理学 X 射线衍射仪 Ultima IV 对陶瓷 的物相结构进行分析, 操作条件为铜靶, $40 \mathrm{kV}$, $40 \mathrm{~mA}$, 扫描速度 $5\left(^{\circ}\right) / \mathrm{min}$, 并用 GSAS 软件精修 XRD 数据。在 $70 \mu \mathrm{L}$ 氧化铝坩埚中, $100 \mathrm{~mL} / \mathrm{min}$ 空 气气氛, 升温速率 $5{ }^{\circ} \mathrm{C} / \mathrm{min}$ 的条件下, 利用梅特勒托利多 TGA/DSC 3+同步热分析仪对生料粉末进行 热重和差示扫描量热测试。采用丹东 Bettersize2000 激光粒度分布仪对生料粉末粒度进行分析。采用 TESCAN 扫描电子显微镜 LYRA3 和牛津 $X$ 射线能 谱仪对陶瓷块体的微观形貌和元素分布进行分析。 采用天津中环电炉可视化形变分析仪 TA-16A01 对 陶瓷块体的烧结收缩过程进行表征。

\section{2 结果与讨论}

\section{1 物相转变和微观形貌}

为了探究升温过程 ZHCYLO 的物相转变过程, 按照一定的工艺(图 1(a))对粉末 A 进行衫烧, 并对 炦烧后的样品进行 XRD 分析。测试结果(图 1(b)) 表明, $1300{ }^{\circ} \mathrm{C}$ 炦烧的样品与煅烧前相比, 物相基 本保持一致; $1400{ }^{\circ} \mathrm{C}$ 煅烧时, 样品各角度范围内 (如 $2 \theta=48^{\circ}$ 和 $57^{\circ}$ 左右) 的衍射峰开始合并 ${ }^{[43]}$; 随着 轱烧温度进一步升高, 主峰/杂峰强度逐渐增强/减 弱, 最终形成单相萤石结构。Gild 等 $^{[41]}$ 采用 $\left(\mathrm{Zr}_{0.25} \mathrm{Hf}_{0.25} \mathrm{Ce}_{0.25} \mathrm{Y}_{0.125} \mathrm{La}_{0.125}\right) \mathrm{O}_{2-\delta}$ 组分合成高熵陶 瓷, 但并未获得单一物相。本研究通过改变元素比 例得到单相萤石结构, 这是因为阳离子的配比直接 影响组分的混合焓, 而混合焓又是影响固溶体形成 的重要因素。过正的混合焓会减小元素之间的混溶 隙, 容易产生元素偏析现象; 而过负的混合焓会诱 导元素之间相互结合, 更容易形成二元化合物。恰 当的阳离子配比会产生接近于 0 的混合焓, 各元素 混乱分布, 陶瓷更容易形成单相结构, 这为非等摩 尔高熵陶瓷提供了更为广阔的发展空间 ${ }^{[43-44]}$ 。对单

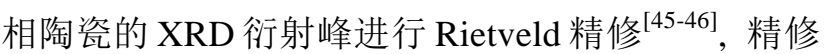
结果如图 2 所示。ZHCYLO 的晶胞参数约为
$0.5387 \mathrm{~nm}$ ，该值处于 $\mathrm{CeO}_{2}$ 晶胞参数 $0.5411 \mathrm{~nm}$ 和 $\left(\mathrm{Zr}_{0.2} \mathrm{Hf}_{0.2} \mathrm{Ce}_{0.2} \mathrm{Ti}_{0.2} \mathrm{Sn}_{0.2}\right) \mathrm{O}_{2}$ 晶胞参数 $0.53512 \mathrm{~nm}$ 之 间 ${ }^{[47]}$, 这与组分的平均离子半径有一定的关系。

为了探索上述 ZHCYLO 是“高摘”或“熵稳定” 的, 将预制的单相陶瓷低温退火 $4 \mathrm{~h}$, 随炉冷却后 对样品进行 XRD测试。从图 3 可以看出, 1000、1200 和 $1400{ }^{\circ} \mathrm{C}$ 退火后的 ZHCYLO 依旧保持着单相萤石 结构, 并没有产生第二相, 证明这种陶瓷是“高熵” 陶瓷 ${ }^{[47-48]}$ 。在以往的高熵氧化物陶瓷中, $\left(\mathrm{Mg}_{0.2} \mathrm{Cu}_{0.2} \mathrm{Ni}_{0.2} \mathrm{Co}_{0.2} \mathrm{Zn}_{0.2}\right) \mathrm{O}$ 和 $\left(\mathrm{Zr}_{0.2} \mathrm{Hf}_{0.2} \mathrm{Ce}_{0.2} \mathrm{Ti}_{0.2} \mathrm{Sn}_{0.2}\right) \mathrm{O}_{2}$ 都是“熵稳定”的, 它们会在某一温度范围内发生可逆
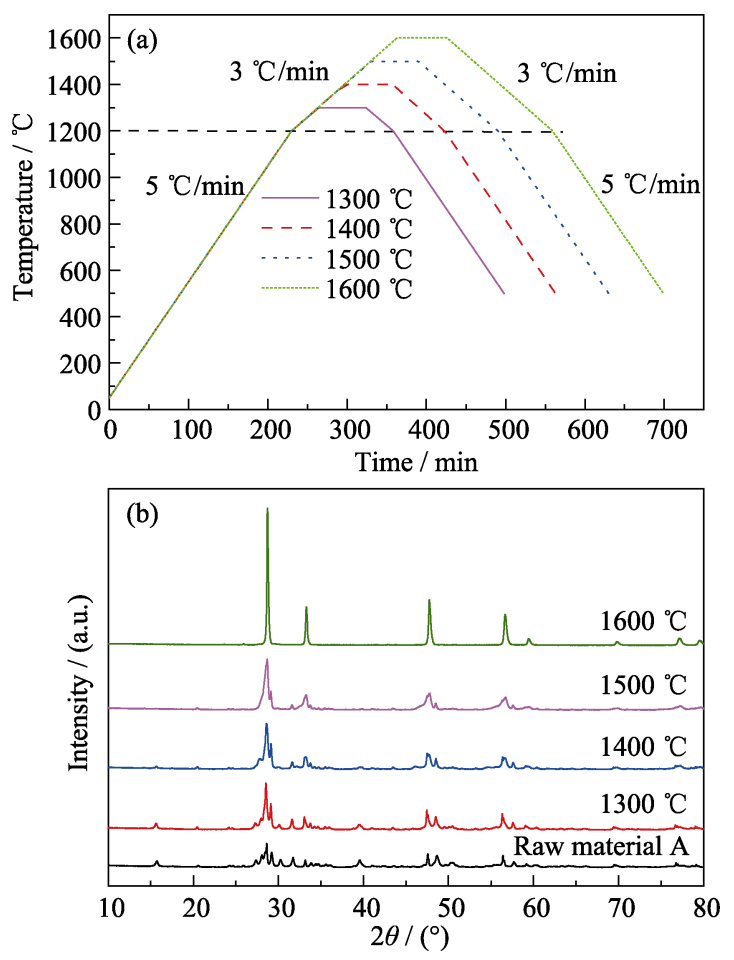

图 1 (a)生料的炦烧工艺和(b)不同温度炦烧生料 A 的 XRD 图谱

Fig. 1 (a) Calcining process of raw materials and (b) XRD patterns of raw material A calcined at different temperatures



图 $21600{ }^{\circ} \mathrm{C}$ 烧结 ZHCYLO 的 XRD 图谱和 Rietveld 精修 拟合

Fig. 2 XRD pattern together with Rietveld fits of the ZHCYLO sintered at $1600{ }^{\circ} \mathrm{C}$ 
的单相-多相转变, 只有在淬冷的条件下, 单相才得 以保存至室温。而本研究采用程序冷却的方式制备 单相 ZHCYLO，有效避免了淬冷工艺在陶瓷表面和 内部产生裂纹, 进而影响材料性能。

图 4 展现了 ZHCYLO 陶瓷的表面形貌和元素分 布，可以观察到样品表面含有许多圆柱形隧道状的 孔洞。在高熵陶瓷中, 阳离子尺寸/质量无序, 增加了 声子散射，而无压烧结多孔萤石结构进一步说明该 陶瓷可能具有隔热材料的潜力 ${ }^{[49-50]}$ 。另外该陶瓷的元 素分布比较均匀, 没有出现明显的偏析团聚现象 ${ }^{[47]}$ 。

\subsection{TG-DSC}

图 5 是生料粉末 A 的 TG-DSC 曲线, 从图中可 以清晰地看到四个失重峰, 分别位于 $80 、 300 、 600$ 和 $1400{ }^{\circ} \mathrm{C}$ 附近。1 号失重峰对应粉体中吸附水的蒸 发。此外, 在球磨制备生料粉末过程中, $\mathrm{Y}_{2} \mathrm{O}_{3}$ 和 $\mathrm{La}_{2} \mathrm{O}_{3}$ 可能发生水化生成 $\mathrm{Y}(\mathrm{OH})_{3}$ 和 $\mathrm{La}(\mathrm{OH})_{3}{ }^{[51-54]}$, 因此 2 号和 3 号失重峰对应于结晶水的蒸发。在图 1(b)中, $1300{ }^{\circ} \mathrm{C}$ 煅烧粉末的物相结构基本与生料保持一致, $1400{ }^{\circ} \mathrm{C}$ 以上炦烧粉末的物相结构逐渐向单相结构 演变, 而 4 号失重峰正好在 $1400{ }^{\circ} \mathrm{C}$, 综合 XRD 和 TG-DSC 结果, 可以推测 4 号失重峰与单相固溶体

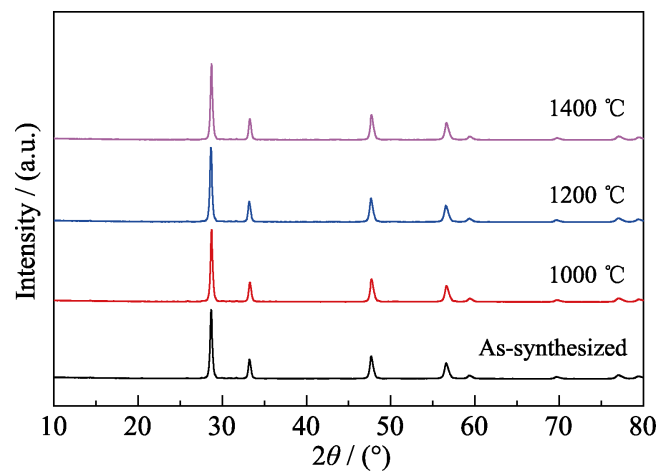

图 3 经不同温度退火的 ZHCYLO 的 XRD 图谱

Fig. 3 XRD patterns of ZHCYLO annealed at different temperatures
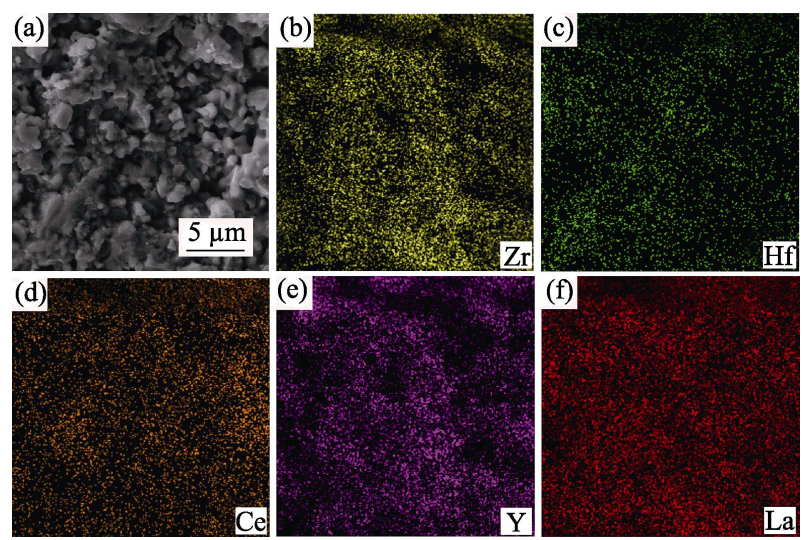

图 4 在 $1600{ }^{\circ} \mathrm{C}$ 下烧结 $1 \mathrm{~h}$ 的 ZHCYLO 的 SEM-EDS 图片

Fig.4 SEM-EDS images of ZHCYLO sintered at $1600{ }^{\circ} \mathrm{C}$ for $1 \mathrm{~h}$

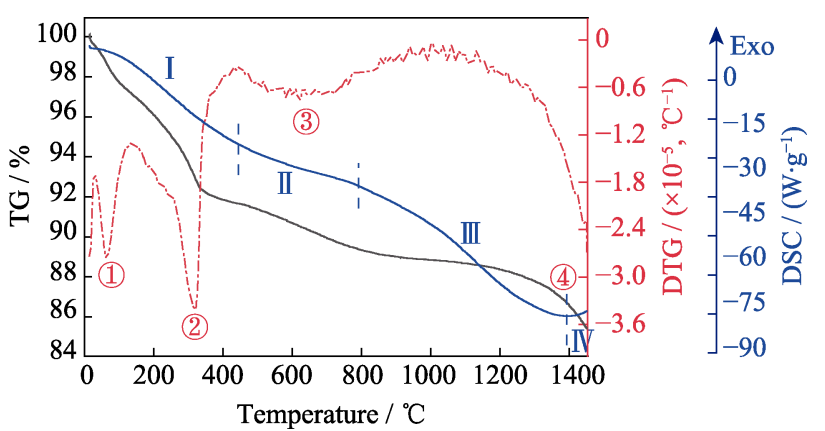

图 5 生料粉末 A 的 TG-DSC 曲线

Fig. 5 TG-DSC curves of raw material A

的形成有着密切联系。DSC 曲线主要分为个四个阶 段：阶段 I 吸附水脱附和部分结晶水蒸发吸收大量 的热; 阶段 II 与粉体中剩余结晶水蒸发吸热有关; 阶段III主要是因为原料中某些氧化物之间发生固溶, 吸收热量; 阶段 IV 有放热的微弱趋势，这可能是由 于陶瓷发生再结晶而逐渐形成茧石结构。

\section{3 球磨工艺对陶瓷烧结的影响}

图 6 为粉末 $\mathrm{A}$ 和 $\mathrm{B}$ 的激光粒度分布曲线, 两者 峰形相近, 均没有表现出单峰趋势, 这是因为各氧 化物原料粒径存在差异。干法球磨工艺作用于粉体 的能量比湿法球磨大得多, 大量机械能转化为颗粒 表面能并产生缺陷, 因此粉末 $\mathrm{B}$ 的粒径整体略小于 粉末 $\mathrm{A}^{[55]}$ 。对比两种生料粉末的 XRD 图谱(图 7(a)) 发现：与 A 粉末相比, B 粉末在“・”标记处的衍射峰有
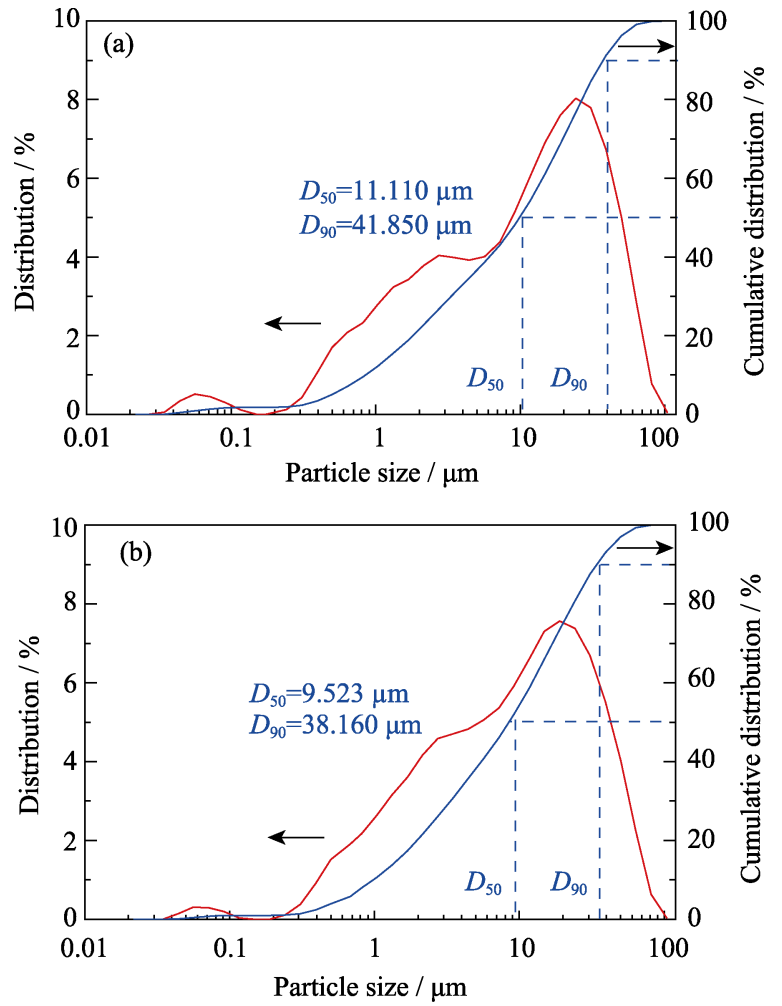

图 6 (a)生料 A 和(b)生料 B 的粒度分布曲线

Fig. 6 Particle size distribution of (a) raw material A and (b) B 
不同程度的角度合并和强度降低现象, 这可能是由于 粉末内部产生少量固态扩散和机械合金化造成的 ${ }^{[56]}$ 。

按照图 1(a)的工艺对粉末 B 进行㷽烧, 煅烧后 样品的 XRD 结果如图 7(b)所示。与生料粉末 A 不 同, 粉末 $\mathrm{B}$ 在 $1300{ }^{\circ} \mathrm{C}$ 煅烧后基本上形成单相萤石 结构; $1500{ }^{\circ} \mathrm{C}$ 煅烧后, 样品衍射峰尖锐, 结晶性强, 不宜再提高㶲烧温度。这可能是由于生料结构和粒 度的协同变化作用使得高熵陶瓷单相转变温度降低 $300{ }^{\circ} \mathrm{C}$ 左右。

图 8 为坏体尺寸随烧结温度变化的曲线和光学

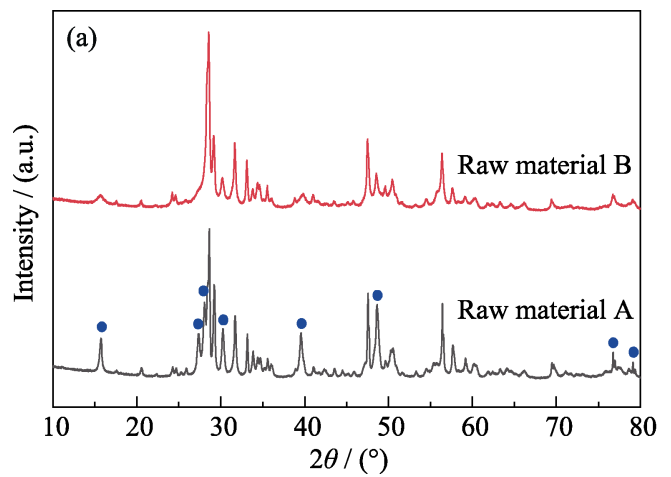

照片, $L / L_{0}$ 为坏体实际尺寸与原始尺寸之比, $1-L / L_{0}$ 为坏体尺寸收缩率。可以看出坏体 $\mathrm{A}$ 在 $1300{ }^{\circ} \mathrm{C}$ 开始 收缩, 在 $1600{ }^{\circ} \mathrm{C}$ 保温 $1 \mathrm{~h}$ 后, 宽和高的收缩率分别为 7.1\%和 $10.1 \%$, 并且持续收缩趋势很大; 在图 8(b, d) 中，坏体 B 同样也在 $1300{ }^{\circ} \mathrm{C}$ 开始收缩，在 $1600{ }^{\circ} \mathrm{C}$ 下保温 $1 \mathrm{~h}$ 后，宽和高尺寸收缩率为 $17.5 \%$ 和 $18.1 \%$, 收缩趋势渐缓直至平稳。综合 $\mathrm{XRD}$ (图 1 和图 7)和 体积收缩(图 8)可知, ZHCYLO 陶瓷在整个烧结过程 中, 固溶反应引起的体积收缩比较微弱, 主要是在 1300 1600 ${ }^{\circ} \mathrm{C}$ 致密烧结产生体积收缩，在该阶段

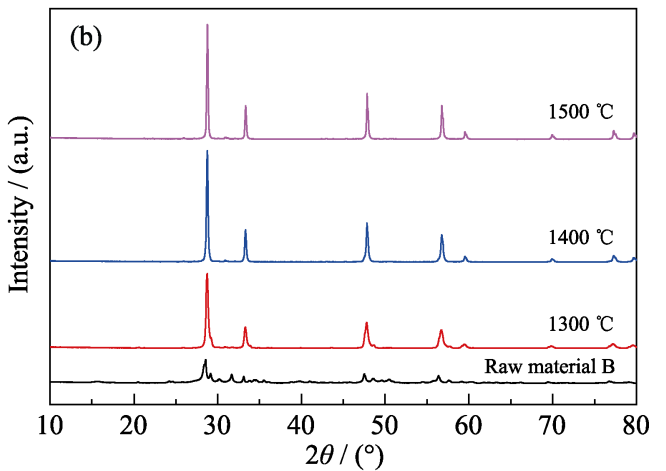

图 7 (a) 生料 A 和 B 的 XRD 图谱对比, (b)不同温度下衤烧生料 B 的 XRD 图谱

Fig. 7 (a) Comparation of XRD patterns from raw material A and B; (b) XRD patterns of raw material B sintered at different temperatures

(a)



(b)



(d)

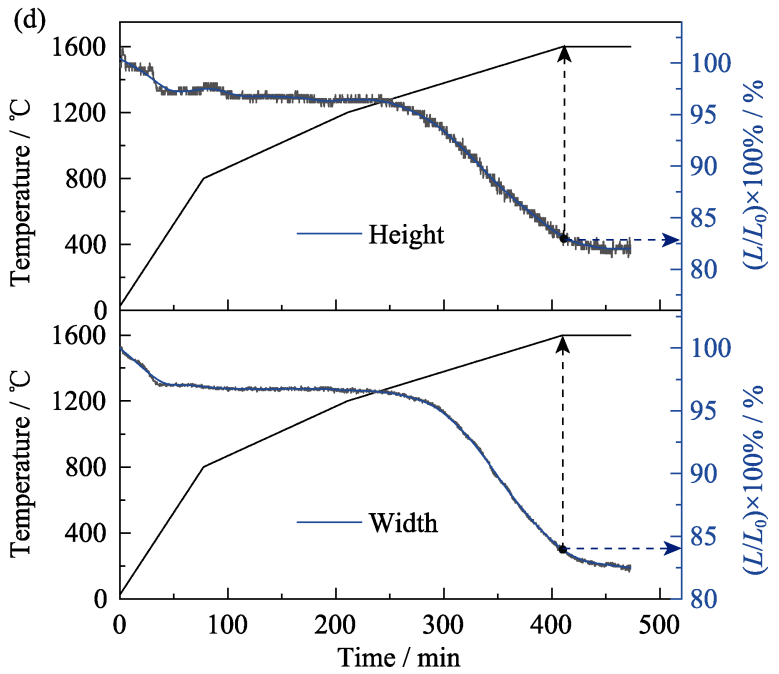

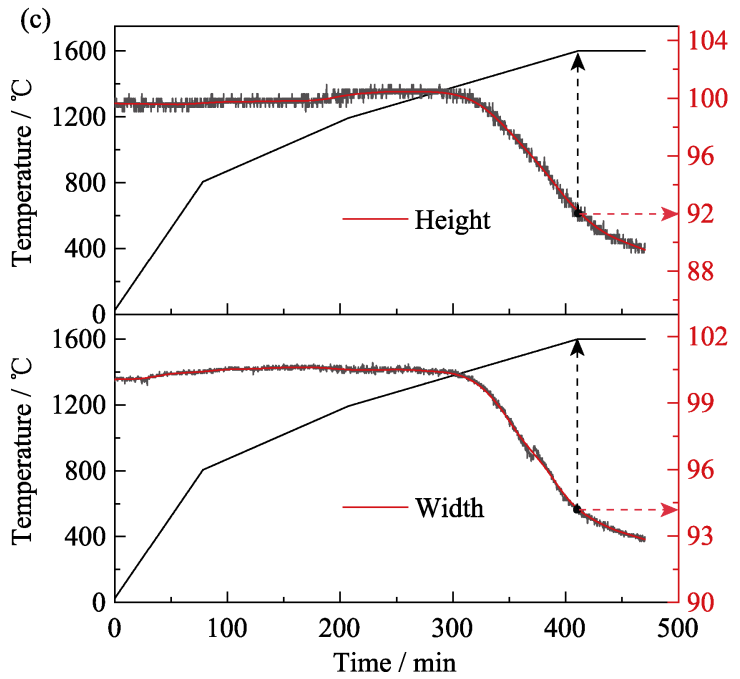

图 8 不同坏体在不同温度下烧结的光学照片以及坏体随时间变化的温度/线性收缩

Fig. 8 Digital camera photographs of different pellets sintered at different temperatures and temper ature/linear shrinkage of different pellets as a function of time 
应该适当降低升温速率，可以防止坏体开裂，提高 坏体质量。

根据陶瓷坏体的尺寸收缩情况可以推测坏体 B 的致密度比 A 高得多。利用 Rietveld 精修的晶胞参 数计算 ZHCYLO 的理论密度 $\rho_{\mathrm{th}}$ 约为 $6.4188 \mathrm{~g} / \mathrm{cm}^{3}$, 随后通过阿基米德排水法测试坏体 $\mathrm{A}$ 和 $\mathrm{B}$ 的致密度 分别为 $82.25 \%$ 和 $93.23 \%$ 。总之, 高熵陶瓷的烧结受 众多因素的影响，除了元素组成、烧结气氛和冷却 速率外 ${ }^{[39]}$, 球磨工艺也是重要影响因素之一。

\section{3 结论}

本研究采用机械球磨和常压烧结的方式, 制备 出一种具有萤石结构的高熵氧化物陶瓷 $\left(\mathrm{Zr}_{1 / 7} \mathrm{Hf}_{1 / 7} \mathrm{Ce}_{1 / 7} \mathrm{Y}_{2 / 7} \mathrm{La}_{2 / 7}\right) \mathrm{O}_{2-\delta}$ 。

1)该陶瓷是一种非等摩尔“高熵”陶瓷，陶瓷内 部金属元素均匀分布, 退火后不会发生单相-多相 转变;

2)常压烧结制备 $\left(\mathrm{Zr}_{1 / 7} \mathrm{Hf}_{1 / 7} \mathrm{Ce}_{1 / 7} \mathrm{Y}_{2 / 7} \mathrm{La}_{2 / 7}\right) \mathrm{O}_{2-\delta}$ 坏 体, 1300 1600 ${ }^{\circ} \mathrm{C}$ 阶段发生物相转变和体积收缩, 此时应放缓升温速率并延长加热时间, 防止坯体 开裂;

3)湿法球磨和干法球磨制备的生料粉末结构和 粒度均不同, 坏体在 $1600{ }^{\circ} \mathrm{C}$ 保温 $1 \mathrm{~h}$ 后, 尺寸收缩 率为 $8.5 \%$ 和 $17.8 \%$, 致密度分别为 $82.25 \%$ 和 93.23\%。不同的球磨工艺会影响陶瓷的烧结性能。

\section{参考文献:}

[1] MIRACLE D B, SENKOV O N. A critical review of high entropy alloys and related concepts. Acta Materialia, 2017, 122: 448-511.

[2] ZHU J M, FU H M, ZHANG F, et al. Synthesis and properties of multiprincipal component AlCoCrFeNiSi ${ }_{x}$ alloys. Materials Science and Engineering: A, 2010, 527(27): 7210-7214.

[3] SZKLARZ Z, LEKKI J, BOBROWSKI P, et al. The effect of SiC nanoparticles addition on the electrochemical response of mechanically alloyed CoCrFeMnNi high entropy alloy. Materials Chemistry and Physics, 2018, 215: 385-392.

[4] TSAI M, WANG C W, TSAI C W, et al. Thermal stability and performance of NbSiTaTiZr high-entropy alloy barrier for copper metallization. Journal of The Electrochemical Society, 2011, 158(11): H1161-H1165.

[5] ROST C M, SACHET E, BORMAN T, et al. Entropy-stabilized oxides. Nature Communications, 2015, 6(1): 8485.

[6] WEI X F, LIU J X, LI F, et al. High entropy carbide ceramics from different starting materials. Journal of the European Ceramic Society, 2019, 39(10): 2989-2994.

[7] JIN T, SANG X H, UNOCIC R R, et al. Mechanochemical-assisted synthesis of high-entropy metal nitride via a soft urea strategy. Advanced Materials, 2018, 30(23): 1707512.

[8] LIU J X, SHEN X Q, WU Y, et al. Mechanical properties of hot-pressed high-entropy diboride-based ceramics. Journal of Advanced Ceramics, 2020, 9(4): 503-510.

[9] QIN Y, LIU J X, LI F, et al. A high entropy silicide by reactive spark plasma sintering. Journal of Advanced Ceramics, 2019, 8(1): 148-152.

[10] CHEN X Q, WU Y Q. High-entropy transparent fluoride laser ceramics. Journal of the American Ceramic Society, 2020, 103(2): 750-756.

[11] ZHANG R Z, GUCCI F, ZHU H Y, et al. Data-driven design of ecofriendly thermoelectric high-entropy sulfides. Inorganic Chemistry, 2018, 57(20): 13027-13033.

[12] DJENADIC R, SARKAR A, CLEMENS O, et al. Multicomponent equiatomic rare earth oxides. Materials Research Letters, 2017, 5(2): 102-109.

[13] MAO A Q, XIANG H Z, ZHANG Z G, et al. Solution combustion synthesis and magnetic property of rock-salt $\left(\mathrm{Co}_{0.2} \mathrm{Cu}_{0.2} \mathrm{Mg}_{0.2} \mathrm{Ni}_{0.2} \mathrm{Zn}_{0.2}\right) \mathrm{O}$ high-entropy oxide nanocrystalline powder. Journal of Magnetism and Magnetic Materials, 2019, 484: 245-252.

[14] XING Q W, XIA S Q, YAN X H, et al. Mechanical properties and thermal stability of (NbTiAlSiZr) $\mathrm{N}_{x}$ high-entropy ceramic films at high temperatures. Journal of Materials Research, 2018, 33(19): 3347-3354.

[15] CHEN L, WANG K, SU W T, et al. Research progress of transition metal non-oxide high-entropy ceramics. Journal of Inorganic Materials, 2020, 35(7): 748-758.

[16] CHEN H, QIU N, WU B Z, et al. Tunable pseudocapacitive contribution by dimension control in nanocrystalline-constructed $\left(\mathrm{Mg}_{0.2} \mathrm{Co}_{0.2} \mathrm{Ni}_{0.2} \mathrm{Cu}_{0.2} \mathrm{Zn}_{0.2}\right) \mathrm{O}$ solid solutions to achieve superior lithium-storage properties. RSC Advances, 2019, 9(50): 28908-28915.

[17] BÉRARDAN D, FRANGER S, DRAGOE D, et al. Colossal dielectric constant in high entropy oxides. Physica Status Solidi, 2016, 10(4): 328-333.

[18] ZHANG J J, YAN J Q, CALDER S, et al. Long-range antiferromagnetic order in a rocksalt high entropy oxide. Chemistry of Materials, 2019, 31(10): 3705-3711.

[19] BéRARDAN D, FRANGER S, MEENA A K, et al. Room temperature lithium superionic conductivity in high entropy oxides. Journal of Materials Chemistry A, 2016, 4(24): 9536-9541.

[20] CHEN H, FU J, ZHANG P F, et al. Entropy-stabilized metal oxide solid solutions as $\mathrm{CO}$ oxidation catalysts with high-temperature stability. Journal of Materials Chemistry A, 2018, 6(24): 11129-11133.

[21] CHEN H, LIN W W, ZHANG Z H, et al. Mechanochemical synthesis of high entropy oxide materials under ambient conditions: dispersion of catalysts via entropy maximization. ACS Materials Letters, 2019, 1(1): 83-88.

[22] SARKAR A, DJENADIC R, WANG D, et al. Rare earth and transition metal based entropy stabilised perovskite type oxides. Journal of the European Ceramic Society, 2018, 38(5): 2318-2327.

[23] PU Y P, ZHANG Q W, LI R, et al. Dielectric properties and electrocaloric effect of high-entropy $\left(\mathrm{Na}_{0.2} \mathrm{Bi}_{0.2} \mathrm{Ba}_{0.2} \mathrm{Sr}_{0.2} \mathrm{Ca}_{0.2}\right) \mathrm{TiO}_{3}$ ceramic. Applied Physics Letters, 2019, 115(22): 223901.

[24] LIU J, REN K, MA C Y, et al. Dielectric and energy storage properties of flash-sintered high-entropy $\left(\mathrm{Bi}_{0.2} \mathrm{Na}_{0.2} \mathrm{~K}_{0.2} \mathrm{Ba}_{0.2} \mathrm{Ca}_{0.2}\right) \mathrm{TiO}_{3}$ ceramic. Ceramics International, 2020, 46(12): 20576-20581.

[25] EDALATI P, WANG Q, RAZAVI-KHOSROSHAHI H, et al. Photocatalytic hydrogen evolution on a high-entropy oxide. Journal of Materials Chemistry A, 2020, 8(7): 3814-3821.

[26] WANG T, CHEN H, YANG Z, et al. High-entropy perovskite fluorides: a new platform for oxygen evolution catalysis. Journal of the American Chemical Society, 2020, 142(10): 4550-4554.

[27] FRACCHIA M, MANZOLI M, ANSELMI-TAMBURINI U, et al. A new eight-cation inverse high entropy spinel with large 
configurational entropy in both tetrahedral and octahedral sites: Synthesis and cation distribution by X-ray absorption spectroscopy. Scripta Materialia, 2020, 188: 26-31.

[28] WANG D, JIANG S D, DUAN C Q, et al. Spinel-structured high entropy oxide (FeCoNiCrMn$)_{3} \mathrm{O}_{4}$ as anode towards superior lithium storage performance. Journal of Alloys and Compounds, 2020, 844: 156158.

[29] MAO A Q, QUAN F, XIANG H Z, et al. Facile synthesis and ferrimagnetic property of spinel $(\mathrm{CoCrFeMnNi})_{3} \mathrm{O}_{4}$ high-entropy oxide nanocrystalline powder. Journal of Molecular Structure, 2019, 1194: 11-18.

[30] WANG J B, STENZEL D, AZMI R, et al. Spinel to rock-salt transformation in high entropy oxides with $\mathrm{Li}$ incorporation. Electrochem, 2020, 1(1): 60-74.

[31] LI F, ZHOU L, LIU J X, et al. High-entropy pyrochlores with low thermal conductivity for thermal barrier coating materials. Journal of Advanced Ceramics, 2019, 8(4): 576-582.

[32] CHEN H, ZHAO Z F, XIANG $\mathrm{H}$ M, et al. High entropy $\left(\mathrm{Y}_{0.2} \mathrm{Yb}_{0.2} \mathrm{Lu}_{0.2} \mathrm{Eu}_{0.2} \mathrm{Er}_{0.2}\right)_{3} \mathrm{Al}_{5} \mathrm{O}_{12}$ : a novel high temperature stable thermal barrier material. Journal of Materials Science \& Technology, 2020, 48: 57-62.

[33] ZHAO Z F, CHEN H, XIANG H M, et al. $\left(\mathrm{La}_{0.2} \mathrm{Ce}_{0.2} \mathrm{Nd}_{0.2} \mathrm{Sm}_{0.2} \mathrm{Eu}_{0.2}\right) \mathrm{PO}_{4}$ : a high-entropy rare-earth phosphate monazite ceramic with low thermal conductivity and good compatibility with $\mathrm{Al}_{2} \mathrm{O}_{3}$. Journal of Materials Science \& Technology, 2019, 35(12): 2892-2896.

[34] CHEN H, XIANG H M, DAI F Z, et al. High entropy $\left(\mathrm{Yb}_{0.25} \mathrm{Y}_{0.25} \mathrm{Lu}_{0.25} \mathrm{Er}_{0.25}\right)_{2} \mathrm{SiO}_{5}$ with strong anisotropy in thermal expansion. Journal of Materials Science \& Technology, 2020, 36: 134-139.

[35] VINNIK D A, TROFIMOV E A, ZHIVULIN V E, et al. The new extremely substituted high entropy (Ba,Sr,Ca,La)Fe $\mathrm{Fe}_{6-x}$ $(\mathrm{Al}, \mathrm{Ti}, \mathrm{Cr}, \mathrm{Ga}, \mathrm{In}, \mathrm{Cu}, \mathrm{W})_{x} \mathrm{O}_{19}$ microcrystals with magnetoplumbite structure. Ceramics International, 2020, 46(7): 9656-9660.

[36] VINNIK D A, ZHIVULIN V E, TROFIMOV E A, et al. Extremely polysubstituted magnetic material based on magnetoplumbite with a hexagonal structure: synthesis, structure, properties, prospects. Nanomaterials (Basel), 2019, 9(4): 559.

[37] SKINNER S J, KILNER J A. Oxygen ion conductors. Materials Today, 2003, 6(3): 30-37.

[38] SACHKOV V I, NEFEDOV R A, AMELICHKIN I V. High entropy oxide systems based on rare earth elements. IOP Conference Series: Materials Science and Engineering, 2019, 597: 012005.

[39] PIANASSOLA M, LOVEDAY M, MCMURRAY J W, et al. Solid-state synthesis of multicomponent equiatomic rare-earth oxides. Journal of the American Ceramic Society, 2020, 103(4): 2908-2918.

[40] SARKAR A, LOHO C, VELASCO L, et al. Multicomponent equiatomic rare earth oxides with a narrow band gap and associated praseodymium multivalency. Dalton Trans., 2017, 46(36): 12167-12176.
[41] GILD J, SAMIEE M, BRAUN J L, et al. High-entropy fluorite oxides. Journal of the European Ceramic Society, 2018, 38(10): 3578-3584.

[42] SHANNON R D. Revised effective ionic radii and systematic studies of interatomic distances in halides and chalcogenides. Acta Crystallographica Section A, 1976, 32(5): 751-767.

[43] WRIGHT A J, WANG Q Y, HUANG C Y, et al. From high-entropy ceramics to compositionally-complex ceramics: a case study of fluorite oxides. Journal of the European Ceramic Society, 2020, 40(5): 2120-2129.

[44] LIU Y C, JIA D C, ZHOU Y, et al. $\mathrm{Zn}_{0.1} \mathrm{Ca}_{0.1} \mathrm{Sr}_{0.4} \mathrm{Ba}_{0.4} \mathrm{ZrO}_{3}$ : a non-equimolar multicomponent perovskite ceramic with low thermal conductivity. Journal of the European Ceramic Society, 2020, 40(15): 6272-6277.

[45] ARTINI C, PANI M, CARNASCIALI M M, et al. Structural features of Sm- and Gd-doped ceria studied by synchrotron X-ray diffraction and $\mu$-Raman spectroscopy. Inorganic Chemistry, 2015, 54(8): 4126-4137.

[46] TOBY B H. EXPGUI, a graphical user interface for GSAS. Journal of Applied Crystallography, 2001, 34(2): 210-213.

[47] CHEN K P, PEI X T, TANG L, et al. A five-component entropy-stabilized fluorite oxide. Journal of the European Ceramic Society, 2018, 38(11): 4161-4164.

[48] DRAGOE N, BéRARDAN D. Order emerging from disorder. Science, 2019, 366(6465): 573.

[49] CHEN H, XIANG H M, DAI F Z, et al. High porosity and low thermal conductivity high entropy $\left(\mathrm{Zr}_{0.2} \mathrm{Hf}_{0.2} \mathrm{Ti}_{0.2} \mathrm{Nb}_{0.2} \mathrm{Ta}_{0.2}\right) \mathrm{C}$. Journal of Materials Science \& Technology, 2019, 35(8): 1700-1705.

[50] WRIGHT A J, WANG Q Y, KO S T, et al. Size disorder as a descriptor for predicting reduced thermal conductivity in mediumand high-entropy pyrochlore oxides. Scripta Materialia, 2020, 181: 76-81.

[51] KURODA Y, HAMANO H, MORI T, et al. Specific adsorption behavior of water on a $\mathrm{Y}_{2} \mathrm{O}_{3}$ surface. Langmuir, 2000, 16(17): 6937-6947.

[52] SPIRIDIGLIOZZI L, FERONE C, CIOFFI R, et al. Entropy-stabilized oxides owning fluorite structure obtained by hydrothermal treatment. Materials, 2020, 13(3): 558-1-12.

[53] CHEN H, ZHAO Z F, XIANG H M, et al. Effect of reaction routes on the porosity and permeability of porous high entropy $\left(\mathrm{Y}_{0.2} \mathrm{Yb}_{0.2} \mathrm{Sm}_{0.2} \mathrm{Nd}_{0.2} \mathrm{Eu}_{0.2}\right) \mathrm{B}_{6}$ for transpiration cooling. Journal of Materials Science \& Technology, 2020, 38: 80-85.

[54] CUI S F, YANG W S, QIAN Z N. Research thermal decomposition fo lanthanum hydroxide by thermogravimetry. Chemical Journal of Chinese University, 1987, 8(3): 271-272.

[55] SURYANARAYANA C. Mechanical alloying and milling. Progress in Materials Science, 2001, 46(1/2): 1-184.

[56] HARRINGTON T J, GILD J, SARKER P, et al. Phase stability and mechanical properties of novel high entropy transition metal carbides. Acta Materialia, 2019, 166: 271-280. 